\title{
Benchmarking of thorium-232 evaluations against spherical transmission and $(n, x n)$ reaction experimental data
}

\author{
S.P. Simakov ${ }^{1, a}$, M.G. Kobozev², A.A. Lychagin², V.A. Talalaev², D.Yu. Chuvilin ${ }^{3}$, and V.M. Maslov ${ }^{4}$ \\ 1 Forschungszentrum Karlsruhe, Institute for Reactor Safety, 76021 Karlsruhe, Germany \\ 2 Institute of Physics and Power Engineering, 249030 Obninsk, Russia \\ 3 RRC "Kurchatov Institute", 123182 Moscow, Russia \\ 4 Joint Institute for Nuclear and Energy Research, 220109 Minsk-Sosny, Belarus
}

\begin{abstract}
The neutron leakage spectra from a thorium sphere of $26 \mathrm{~cm}$ outer and $6 \mathrm{~cm}$ inner diameters with a central T-D and ${ }^{252} \mathrm{Cf}$ neutron sources measured at the Institute of Physics and Power Engineering as well as available in the literature measured differential cross sections for $\mathrm{Th}(\mathrm{n}, \mathrm{xn})$ reaction at $14 \mathrm{MeV}$ were used for benchmarking of the evaluated cross sections from ENDF (versions B-VII.0 and B-VI.8), JEFF-3.1 and Maslov'07 libraries. It was finally concluded that while it is difficult to prefer any of these libraries relying on spherical benchmarks validation results, $\mathrm{Th}(\mathrm{n}, \mathrm{xn})$ differential cross sections are certainly better reproduced by ENDF/B-VII.
\end{abstract}

\section{Introduction}

Thorium is a key element in the Th- $\mathrm{U}$ fuel cycle considered as an alternative nuclear technology option since the advent of commercial nuclear reactor till nowadays. This stimulated the evaluation and experimental validation of the neutron cross sections during a few decades. The most recent one was a Coordinated Research Program "Evaluated nuclear data file for Th-U fuel cycle" coordinated by the IAEA Nuclear Data Section in the period $2002-2006$ [1], which produced a new ${ }^{232}$ Th evaluation for ENDF/B-VII.0 library [2] and stimulated upgrade of previous ones.

This paper presents the neutron leakage spectra from thorium shell with $\mathrm{T}(\mathrm{d}, \mathrm{n})$ and ${ }^{252} \mathrm{Cf}$ neutron sources measured and completely analysed at IPPE in the period $1987-1992$ using evaluated data available then [3-5] (up to now this is the only spherical benchmark for Th). In the present study these experimental data and $\mathrm{Th}(\mathrm{n}, \mathrm{xn})$ double differential cross sections (DDX) at $14 \mathrm{MeV}$ available in the literature [6,7] are used for validation of the new evaluations from ENDF/B (versions VII.0 and VI.8), JEFF-3.1 [8] and "Maslov'07" (version Jan. 2007) [9] libraries.

\section{Experiment}

The measurements of the neutron spectra leaking from the Th sphere have been carried out on the fast neutron spectrometer of IPPE by time of flight technique (TOF) with pulsed neutron generator KG-0.3 producing $14 \mathrm{MeV}$ neutrons via $\mathrm{T}(\mathrm{d}, \mathrm{n})$ reaction and ${ }^{252} \mathrm{Cf}$ ionization chamber emitting fission neutrons [3-5]. Both benchmarks employed one pure metallic thorium sphere having outer/inner radii of $13.0 / 3.0 \mathrm{~cm}$ (wall thickness $10 \mathrm{~cm}$ or 2.2 mean free path for $14 \mathrm{MeV}$ neutrons) and $\phi$ $5.0 \mathrm{~cm}$ hole for the neutron sources accommodation.

\footnotetext{
${ }^{a}$ Presenting author, e-mail: simakov@irs.fzk.de
}

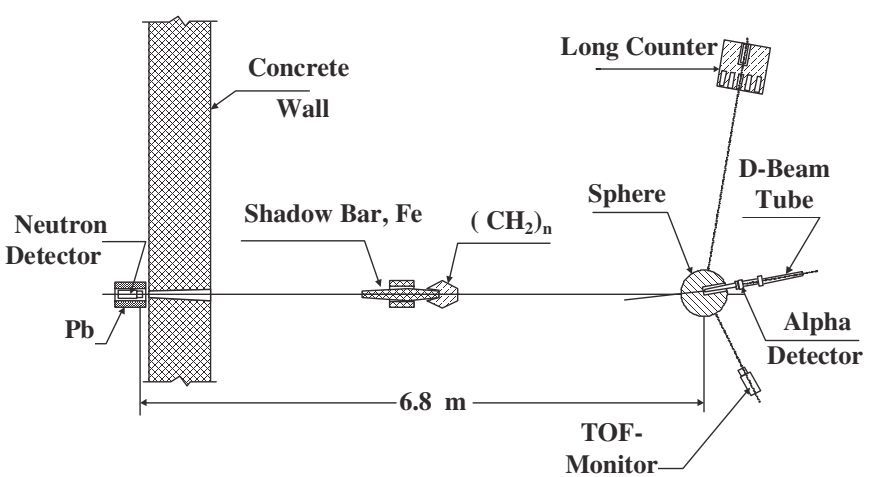

Fig. 1. The experimental set up for the neutron leakage spectra measurement from Th sphere with $\mathrm{T}(\mathrm{d}, \mathrm{n})$ source.

In the experiment with $\mathrm{T}(\mathrm{d}, \mathrm{n})$ neutron source the neutron scintillation detector was located at $682.5 \mathrm{~cm}$ flight path from the Th sphere in a lead shield behind concrete wall at the angle $8^{\circ}$ relative to the deuteron beam (fig. 1). The yield of $14 \mathrm{MeV}$ neutrons was determined by recording the associated $\alpha$-particles spectrum with a calibrated silicon detector installed in deuteron drift tube at $175^{\circ}$. The background neutron spectra were measured with a $1.3 \mathrm{~m}$ long iron/polyethylene shadow bar.

Experiment with ${ }^{252} \mathrm{Cf}$ neutron source has been carried out with a fast ionization chamber (fig. 2), having $34 \mathrm{~mm}$ diameter and $120 \mathrm{~mm}$ length and filled with $\mathrm{Ar}-\mathrm{CO}_{2}$ gas. The one disc electrode had ${ }^{252} \mathrm{Cf}$ layer with intensity of $4 \times 10^{5} \mathrm{n} / \mathrm{s}$. The output pulses of the chamber, after cutting those produced by $\alpha$-particles, fed the stop inputs of the TOF measuring modules and counters to monitor the total number of fission events during the experiment.

The scintillator detector was located at $4.0 \mathrm{~m}$ flight path from the sphere center. The angle between the axis of the hole in the sphere and the direction to the detector was $135^{\circ}$ to reduce the influence of the streaming of source neutrons through the hole. For background measurements an iron shadow bar was installed between the detector and Th sphere. 


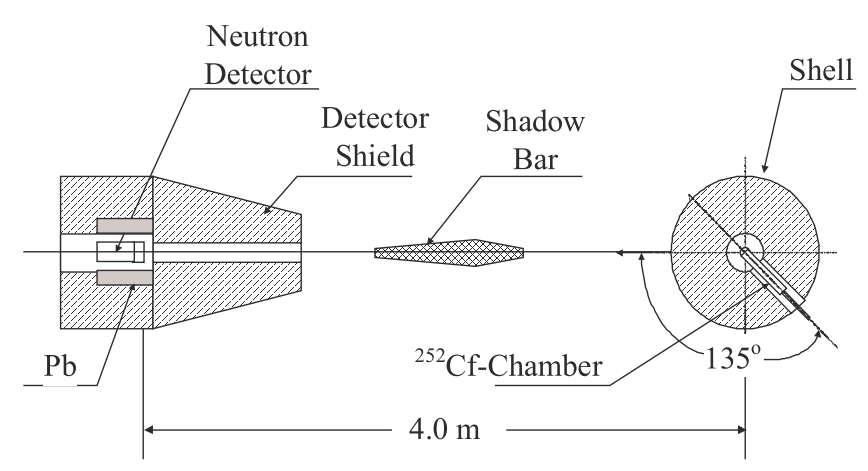

Fig. 2. The experimental set up for the neutron leakage spectra measurement from $\mathrm{Th}$ sphere with ${ }^{252} \mathrm{Cf}$ source.

The efficiency of the detector was measured up to $10 \mathrm{MeV}$ employing the same ${ }^{252} \mathrm{Cf}$ neutron source by removing thorium sphere. This results in partial elimination of the experimental uncertainties. For the determination of the detector efficiency a standard ${ }^{252} \mathrm{Cf}$ spontaneous fission spectrum [10] was used from threshold up to $10 \mathrm{MeV}$ and associated $\alpha$-particle method at $14 \mathrm{MeV}$.

\section{MCNP model for spherical benchmarks}

The calculation with the evaluated data files by means of Monte Carlo transport code MCNP [11] needs correct representation of the most essential details of the experiment. For this purpose we elaborated the input file which includes the thorium shell with a hole for neutron source accommodation $[3-5,12]$. Both the thick TiT target neutrons yield and energy were represented as dependent on emission angle. The target holder was simulated in details to account for the TiT target neutrons scattering leading to the attenuation of $14 \mathrm{MeV}$ neutrons (drop at $90^{\circ}$ ) and generation of the neutrons with lower energies. In the case of ${ }^{252} \mathrm{Cf}$ neutron source the energy distribution was taken from evaluation [10]. Since the fission chamber was made from thin and low mass materials, their distortion on the spectrum was shown to be less than $4 \%[5,12]$. Moreover since leakage spectra and detector efficiency were measured in the same experimental conditions (just removing Th sphere) the influence of chamber materials, scattering by air and collimator were eliminated during data processing.

The measured time of arrival neutron spectrum was converted to the energy one supposing a strict relation between the neutron energy $E$, the sample-detector distance $L$ and the flight time $t$. This is fulfilled only approximately in the case of bulk benchmark and, as was firstly shown for V, Fe, and $\mathrm{LiPb}$ spheres [12], results to some effects such as the shifting of resonances visible below $1 \mathrm{MeV}$ to low energies. To prove this for the Th sphere, we re-calculated (now using ENDF/BVII.0) two leakage energy spectra: L(E) - the MCNP energy tally; and $\mathrm{L}_{\mathrm{t}}(\mathrm{E})$ - time tally converted to energy spectrum. Fig. 3 shows that neutron travelling in Th sphere results to $\approx 200 \mathrm{keV}$ broadening of $14 \mathrm{MeV}$ peak and shifting to the lower energies by $\approx 100 \mathrm{keV}$. Since TOF correction does not exceed $2 \%$ and leakage spectra has no resonance structure, we multiply the measured spectra by $\mathrm{L}(\mathrm{E}) / \mathrm{L}_{\mathrm{t}}(\mathrm{E})$ and propose to

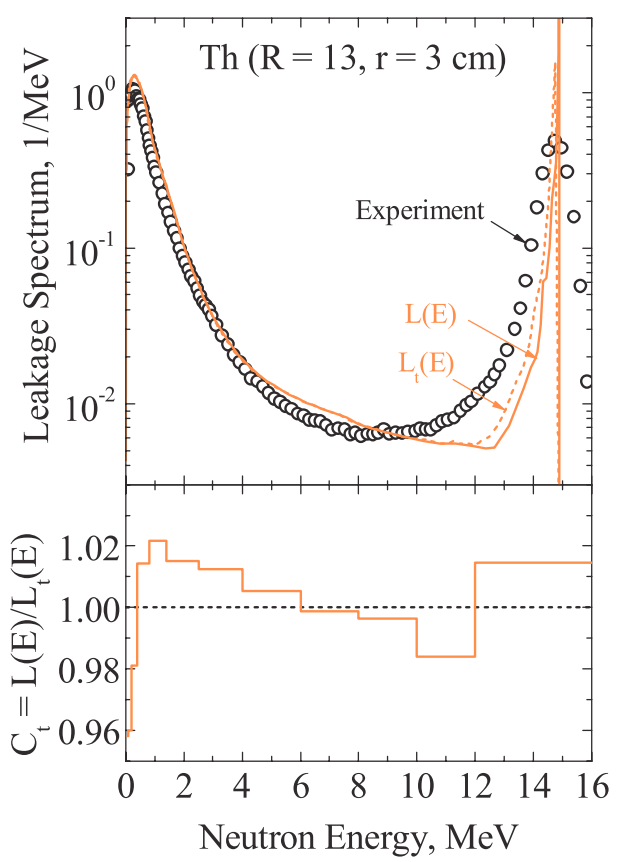

Fig. 3. Upper: neutron leakage spectra measured (circles) and calculated from the MCNP energy (solid) and time of arrival (dashed) tallies using ENDF/B-VII.0. Bottom: TOF correction for Th shell obtained from two calculated spectra.

use the MCNP energy tally for comparison with experimental data.

For an adequate comparison of measurements with transport calculations, the MCNP output leakage energy spectrum needs to be convoluted with the spectrometer response function, accounting for detector energy resolution and neutron interaction with the shielding collimator [12]. Since it is important practically only for the $14 \mathrm{MeV}$ peak we propose to use the measured neutron spectra from bare D-T neutron source (fig. 4). In such approach the subtraction of the estimated spectrum of the neutrons inelastically scattered on the tritium target holder is needed, since they irradiate the inner surface of the shell and hence need to be simulated by including the target assembly in the MCNP input file. The shape of the residual bare source spectrum is formed (as well as a leakage one) by the d-beam pulse time profile and time resolution of spectrometer $(\approx 3-4 \mathrm{~ns})$ that finally determine the $14 \mathrm{MeV}$ peak width (FWHM $\approx 1 \mathrm{MeV}$ at flight path $682 \mathrm{~cm}$ and $\approx 2.5 \mathrm{MeV}$ at $377 \mathrm{~cm}$ ) and by the neutron scattering on the collimator walls that contributes to the low energy asymmetric tail of the $14 \mathrm{MeV}$ peak (fig. 4). We applied such response function for the $12-15 \mathrm{MeV}$ neutrons, whereas below this energy we used a Gaussian with width calculated from the total spectrometer time resolution.

\section{Evaluated data validation}

The transport calculations have been performed by the MCNP code [11] using ENDF/B (versions VII.0 and VI.8) [2], JEFF3.1 [8] and Maslov'07 [9] evaluations for ${ }^{232} \mathrm{Th}$. The experimental and calculated energy distributions of neutrons (and 


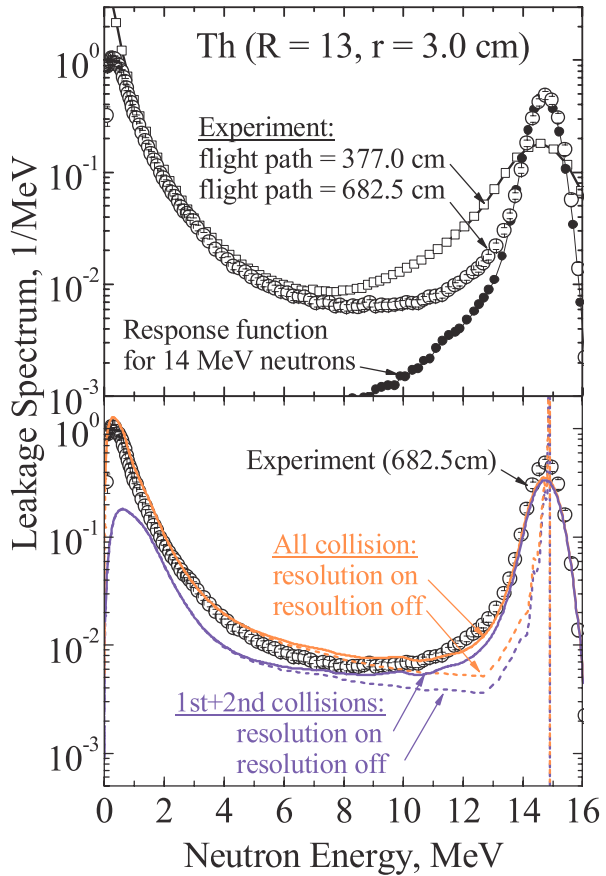

Fig. 4. Measured neutron leakage spectra (open symbols) and spectrometer response function (closed circles). Calculations (ENDF/BVII): total and first two collisions neutron spectra with (solid) and without (dashed) folding with spectrometer response function.

its ratios for indicated energy intervals) leaking from thorium sphere with $\mathrm{T}(\mathrm{d}, \mathrm{n})$ and ${ }^{252} \mathrm{Cf}$ neutron sources are shown in figures 5 and 6 . For the $14 \mathrm{MeV}$ pulsed sphere it is seen that all libraries underestimate by $20 \%$ the attenuation of source neutrons but similarly overestimate the neutrons with lower energies. For the ${ }^{252} \mathrm{Cf}$ source the underestimation for energy range 2 to $6 \mathrm{MeV}$ is also observed for all libraries, the $\mathrm{ENDF} / \mathrm{B}$ being the closer one. In general, it is difficult to give a preference for any of the tested libraries (it depends on energy bin under consideration).

For the $14 \mathrm{MeV}$ neutron source we also estimated the contribution of the first and second collisions to the leakage spectrum. Both have a similar energy shape and magnitude, that means the most neutrons undergoing to collide second time in the shell still have the energy $14 \mathrm{MeV}$. On other hand, as figure 4 shows, the sum of the fist two collisions dominates in the energy range above $2 \mathrm{MeV}$. It means the energy-angular distributions of secondary neutrons from $\mathrm{Th}(\mathrm{n}, \mathrm{xn})$ reactions has to affect on the shape of leakage spectrum.

The evaluated energy differential (angular integrated) cross section for $\mathrm{Th}(\mathrm{n}, \mathrm{xn})$ reaction are compared with available experimental data $[6,7]$ in figure 7 . It might be noted that the ENDF/B-VI.8 and especially the latest version VII.0 better agrees with them reasonably reproducing $8-12 \mathrm{MeV}$ energy interval represented by inelastic scattering to the continuum.

Figure 8 shows the angular distributions of neutrons from $\mathrm{Th}(\mathrm{n}, \mathrm{xn})$ reaction integrated over the indicated secondary neutrons energy bins. It is noted that ENDF/B-VII.0 file is the only one which does predict angular anisotropy of the secondary neutrons and reasonably agrees with measured angular trend.

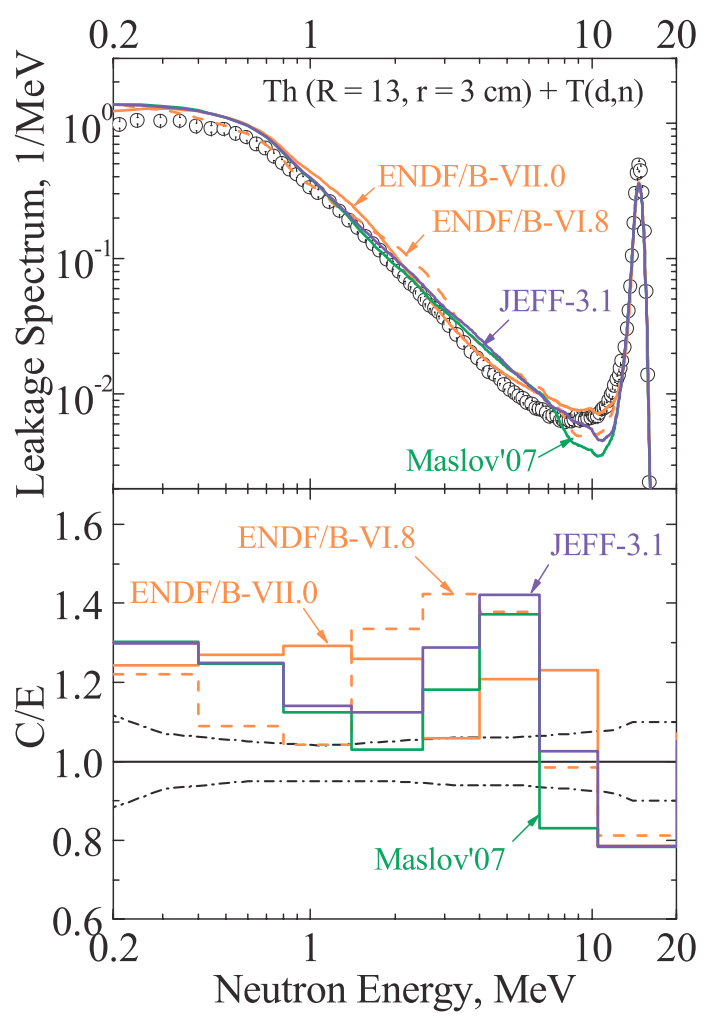

Fig. 5. Neutron leakage spectrum and C/E ratio for Th sphere with $\mathrm{T}(\mathrm{d}, \mathrm{n})$ neutron source. Dashed curves indicate uncertainty corridor.

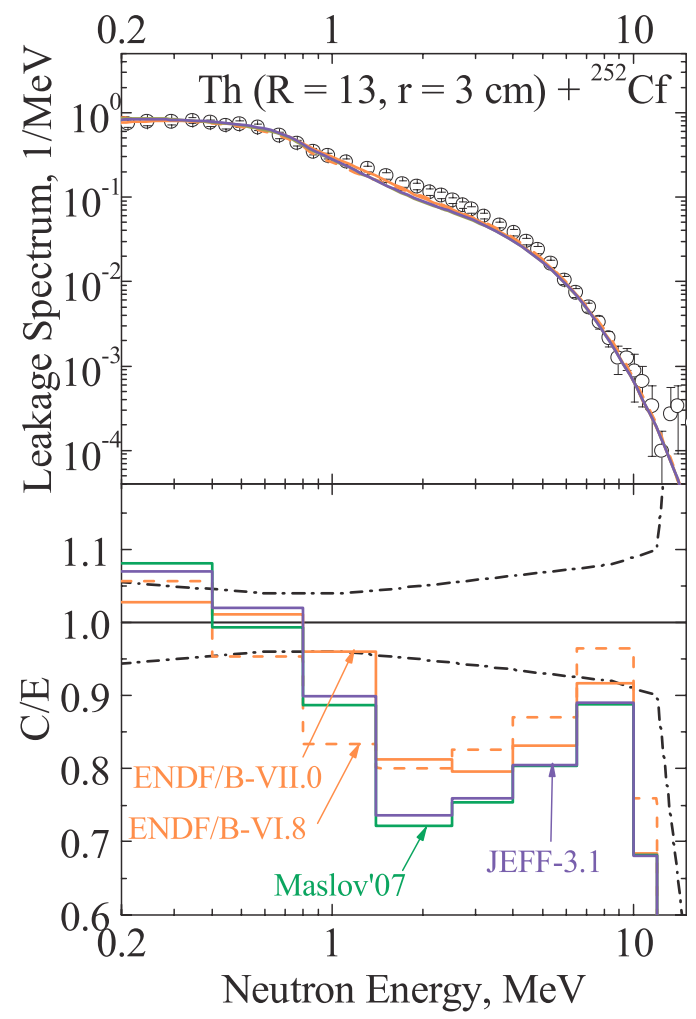

Fig. 6. Neutron leakage spectrum and $\mathrm{C} / \mathrm{E}$ ratio for Th sphere with ${ }^{252} \mathrm{Cf}$ neutron source. Dashed curves indicate uncertainty corridor. 


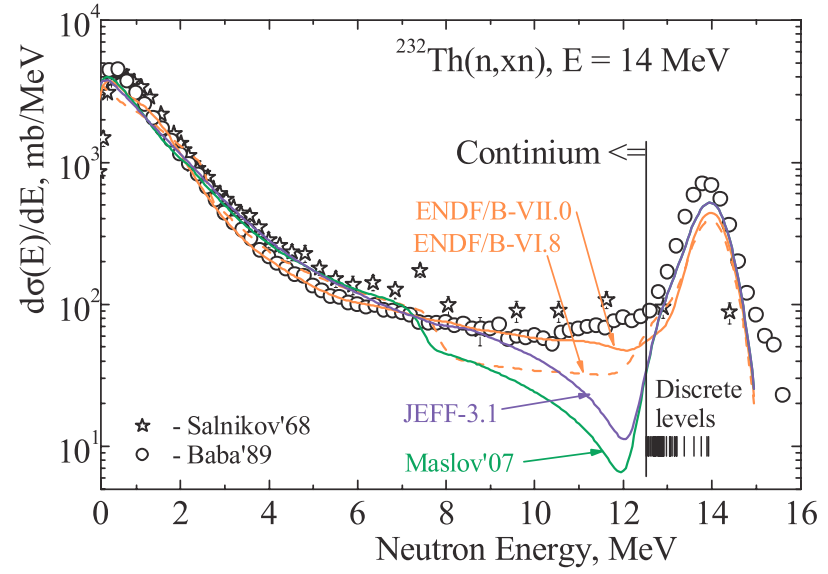

Fig. 7. Energy differential neutron emission cross sections for $\mathrm{Th}(\mathrm{n}, \mathrm{xn})$ reaction at $14 \mathrm{MeV}$. Experiment: $\star$ - O. Salnikov et al. [6], $\bigcirc-$ M. Baba et al. [7]. Evaluated data from libraries ENDF/B-VII.0, JEFF-3.1 and Maslov'07.

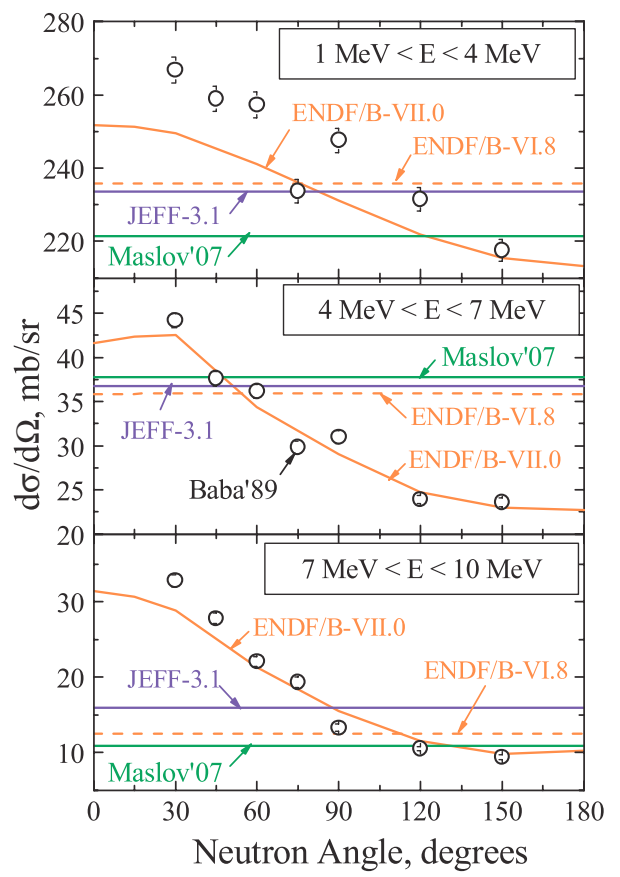

Fig. 8. Angular differential cross sections for $\mathrm{Th}(\mathrm{n}, \mathrm{xn})$ reaction at $14 \mathrm{MeV}$ incident energy and integrated over indicated secondary neutrons energy bins: $\bigcirc$ - experiment of M. Baba et al. [7], curves evaluations.

It is worthwhile to note that calculation of $\mathrm{Th}(\mathrm{n}, \mathrm{xn})$ energy-angular differential distributions has been done by MCNP code using the same ACE files as for the transport calculations. For this we used geometry model representing the measurements of DDX but with very thin wall sample to avoid the multiple neutron scattering. Such approach reproduces the experimental solid angles and energy resolution and avoids handling the data in ENDF format.

\section{Conclusion}

The neutron leakage spectra from a thorium sphere measured several years ago with $\mathrm{T}(\mathrm{d}, \mathrm{n})$ and ${ }^{252} \mathrm{Cf}$ neutron sources were used for the validation of the evaluated neutron cross sections from ENDF/B-VII.0, ENDF/B-VI.8, JEFF-3.1 and Maslov'07 libraries. The spectral deviations were found not to exceed $20-40 \%$ and have a different sign for the different secondary neutrons energy bins. The comparison with $\mathrm{Th}(\mathrm{n}, \mathrm{xn})$ double differential cross sections at $14 \mathrm{MeV}$ has found that ENDF/BVII.0 does better represent the energy distribution of the secondary neutrons and is the only library which predicts its angular anisotropy.

The results of a similar analysis for uranium [13] have shown that the latest ${ }^{238} \mathrm{U}$ evaluations such as ENDF/B-VII agree with measured data typically within $10 \%$, which is practically equal to experimental uncertainties.

\section{References}

1. IAEA Coordinated Research Program - Evaluated nuclear data file for Thorium-Uranium fuel cycle, see http://wwwnds.iaea.org/Th-U/

2. M.B. Chadwick, P. Oblozinsky et al., Nucl. Data Sheets 107, 2931 (2006).

3. S.P. Simakov, B.V. Devkin, M.G. Kobozev et al., Neutron leakage spectra from $\mathrm{Be}, \mathrm{Al}, \mathrm{Fe}, \mathrm{Ni}, \mathrm{Pb}, \mathrm{LiPb}, \mathrm{Bi}, \mathrm{U}$ and $\mathrm{Th}$ with $T(d, n)$ and ${ }^{252} C f$ neutron sources, in Fusion Technology (Elsevier, 1993), Vol. 2, p. 1489.

4. S.P. Simakov, A.A. Androsenko et al., Neutron leakage spectra from $U$ and Th spheres with Cfneutron source, Report ZFK-646, Dresden, 1988, p. 111.

5. S.P. Simakov et al., $14 \mathrm{MeV}$ Facility and Research in IPPE, Report INDC(CCP)-351, IAEA, Vienna, 1993; http://wwwnds.iaea.org/reports-new/indc-reports/indc-ccp/indc-ccp0351.pdf

6. O.A. Salnikov, N.I. Fetisov, G.N. Lovchikova et al., Izv. Rossiiskoi Akademii Nauk, Ser. Fiz. 32, 653 (1968).

7. M. Baba, H. Wakabayshi et al., Report INDC(JPN)-129, IAEA, Vienna, 1989.

8. A.J. Koning et al., The JEFF evaluated nuclear data project (these proceedings).

9. V. Maslov, Updated the Minsk Th-232 evaluation on February 2007; http://www-nds.iaea.org/Th-U/

10. W. Mannhart, Evaluation of the Cf-252 fission neutron spectrum between 0 and $20 \mathrm{MeV}$, in Report IAEA-TECDOC-410, IAEA, Vienna, 1987, p. 158.

11. X-5 Monte Carlo Team, MCNP-A General Monte Carlo NParticle Transport Code, version 5, Report LA-UR-03-1987, Los Alamos (2005).

12. S.P. Simakov et al., Benchmarking of evaluated nuclear data for vanadium by a $14 \mathrm{MeV}$ spherical shell transmission experiment, Report INDC(CCP)-417, 1998;

http://www-nds.iaea.org/reports-new/indc-reports/indcccp/indc-ccp-0417.pdf

13. S.P. Simakov et al., Benchmarking of Uranium-238 Evaluations against Spherical Transmission and (n,xn)-reaction Experimental Data, Proc. of ND2004, Santa Fe, Sept. 2004, AIP 769 (2005), p. 67. 